\title{
Burst-Mode FEC Performance for PON Upstream Channels with EDFA Optical Transients
}

\author{
Nicola Brandonisio, Daniel Carey, Stefano Porto, Giuseppe Talli, and Paul D. Townsend \\ Tyndall National Institute, \\ University College Cork, \\ Cork, Ireland \\ nicola.brandonisio@tyndall.ie
}

\begin{abstract}
The performance of forward error correction (FEC) based on Reed-Solomon coding is analyzed experimentally for a burst-mode upstream channel within a passive optical network (PON) testbed. During this analysis, the upstream FEC performance is impaired by inducing correlated and localized errors within the burst through the injection of optical transients. These transients emulate the optical signal variation associated with the add-and-drop events of wavelength channels within a long-reach optical link based on a chain of erbium doped fiber amplifiers (EDFAs). The robustness of the FEC has been analyzed by measuring the post-FEC bit error rate (BER) as a function of the amplitude of the emulated transients and their delay with respect to the transmitted bursts. A margin of approximately $4 \mathrm{~dB}$ is demonstrated for the transient amplitude before the FEC degradation. Furthermore, while the post-FEC BER is strongly degraded by the emulated transients, the preFEC BER stays below the FEC threshold, demonstrating the importance of measuring the post-FEC BER in order to correctly characterize the FEC performance in PON upstream channels affected by optical transients.
\end{abstract}

Keywords-forward error correction; passive optical networks; burst-mode transmission; field-programmable gate arrays; erbium doped fiber amplifiers; optical transients.

\section{INTRODUCTION}

Long-reach passive optical networks (PONs) have been recently demonstrated capable of providing cost-efficient and flexible solutions for service convergence within the access and metro layers of the Internet infrastructure [1-5]. Different PON architectures have been proposed according to the geographical distribution of the customers which are covered by the network. In particular, a PON architecture based on a single amplifier node (AN) has been demonstrated in $[3,4]$ to provide efficient solutions for densely populated metropolitan areas. Moreover, a PON architecture based on chains of optical ANs, including erbium doped fiber amplifiers (EDFAs), has been demonstrated in [5] to deliver efficient solutions for sparsely populated rural areas.

In all long-reach PON architectures, burst-mode forward error correction (FEC) plays a crucial role for the implementation of the upstream channels within the stringent optical power budget prescribed by the current PON standards

Financial support from Science Foundation Ireland (SFI) (grants 12/IA/1270, 12/RC/2276, and 10/CE/I1853), and EU FP7 project DISCUS (grant CNECT-ICT- 318137) is gratefully acknowledged.
$[6,7]$. In order to achieve the desired performance of the burstmode FEC each network component should be carefully designed. In fact, burst-mode FEC can be affected by strongly correlated and localized errors within the burst, which can be introduced by transient behaviour of the network components.

In this work Reed-Solomon (RS) coding is employed for implementing the burst-mode FEC in accordance with the recommendations of the current PON standards [6,7]. The theoretical performance of RS-based FEC is obtained when errors are uniformly distributed within the burst. However, the PON upstream links are characterized by several sources of impairments which can cause strongly correlated and localized errors within the burst. These sources include the electrical and optical turn-on transients of the transmitter or the distortion potentially introduced by the gain setting of the electrical amplifier in the burst-mode receiver. An example of this concept has been demonstrated in [8] for a state-of-the-art long-reach PON testbed based on a single AN. In this case, the burst-mode FEC performance has been strongly degraded by inducing correlated and localized errors within the burst through the transient behaviour of a clock-and-data recovery unit. Other examples of burst-mode FEC performance degraded by correlated errors can be found in [9] for a PON upstream channel and in [5] for a long-reach PON testbed composed of a chain of EDFA-based ANs. However, in the investigation presented in [5], the burst-mode FEC performance has been only marginally degraded by introducing optical transients within the burst through on-off switching of several wavelength channels.

This paper aims to extend the work presented in [5] by analysing the burst-mode FEC performance with respect to the amplitude of optical transients which can arise in long-reach PONs that employ chains of EDFA-based ANs, when wavelength channels are added and dropped. In this work, these optical transients have been emulated within a simplified testbed in order to adjust the amplitude of the optical transients and their delay with respect to the optical bursts. This analysis has led to determine a margin of approximately $4 \mathrm{~dB}$ for the amplitude of these optical transients before the FEC degradation. In this work the importance of measuring the post-FEC bit error rate (BER) is also demonstrated for correctly evaluating the burst-mode FEC performance in PON upstream links affected by optical transients. 


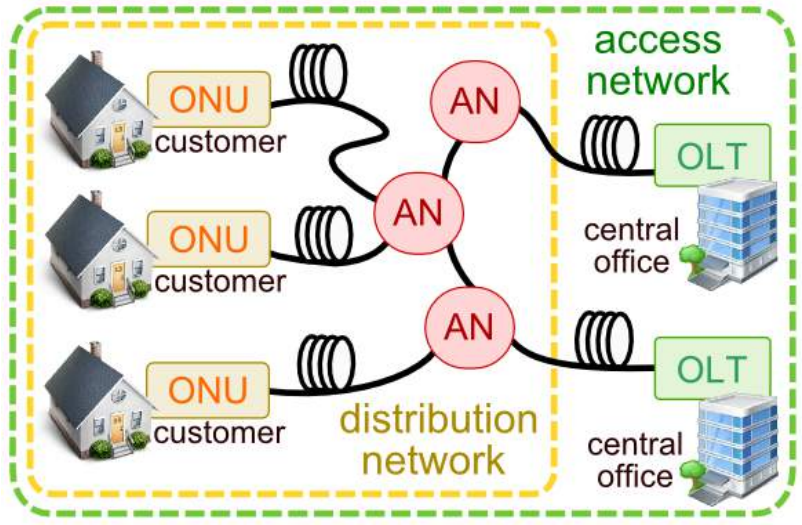

Fig. 1. Long-reach PON based on a chain of amplifier nodes.

This paper is organized as follows. In Section II, the PON testbed is presented with the description of the implemented network components. The technique used for emulating the optical transients is also described. In Section III, the FEC performance within this PON setup is experimentally investigated showing how the measured FEC performance can be degraded by the emulated optical transients. In this way, the robustness of the burst-mode FEC is evaluated with respect to these transients. Finally, in Section IV the conclusions of this work are presented.

\section{PASSive OpticAl Network TeStBed}

The long-reach PON access system considered in this work is based on the three main components schematically represented in Fig. 1: the optical network units (ONUs), the optical line terminals (OLTs), and the optical distribution network (ODN) [1-3]. The ONUs are used by the customers to access the PON infrastructure and are connected to a chain of EDFA-based ANs through the ODN, as described in [5]. The OLTs are located at the central offices and are connected to the ANs through the backhaul links, enabling the connection between the access network and the main Internet infrastructure.

This long-reach PON architecture has been emulated by the testbed used in this work. As shown in Fig. 2, two ONUs and one OLT have been fully developed in hardware using Xilinx Virtex-7 field-programmable gate arrays (FPGAs) on the VC709 development boards. Each ONU employs a semiconductor optical amplifier (SOA) for carving the optical bursts, which are transmitted and received using a standard small form-factor pluggable (SFP+) transceiver. These SFP+ transceivers are tunable across 100 wavelength channels with $50 \mathrm{GHz}$ spacing within the C-band and have a transmission rate of $10 \mathrm{~Gb} / \mathrm{s}$. The upstream bursts generated by these two ONUs are time-multiplexed using a synchronization protocol implemented through the downstream data and managed by the OLT. The ONUs have been calibrated in order to transmit bursts with approximately the same optical power close to $+1 \mathrm{dBm}$. An SFP + transmitter is also used by the OLT for generating the downstream data. The upstream burst traffic is received by the OLT using a linear burst-mode receiver (LBMRx), which is followed by a clock-and-data recovery (CDR) unit [10,11].
Real-time $10 \mathrm{~Gb} / \mathrm{s}$ burst-mode FEC has been implemented on the FPGAs using the RS $(248,216)$ coding technique. This algorithm encodes a block of 216 data symbols into a block of 248 symbols by adding 32 check symbols, where each symbol is a group of 8 bits. When a block of 248 symbols is received, the $\operatorname{RS}(248,216)$ algorithm uses the 32 check symbols for correcting up to 16 corrupted symbols within the received block. This algorithm leads to a post-FEC BER lower than 1e12 for a pre-FEC BER lower than 1.1e-3, assuming that the errors are uncorrelated and uniformly distributed within the burst. The pre-FEC BER value of 1.1e-3 is defined as the FEC threshold. Here, the optical power received by the OLT has been adjusted using a variable attenuator in order to obtain a pre-FEC BER for both ONUs close to the FEC threshold. This situation represents the worst case scenario for the FEC performance and leads to a conservative estimation of the impact of the emulated optical transients on the FEC.

As shown in the PON testbed depicted in Fig. 2, the power transients within the upstream burst traffic are emulated by driving an SOA with a waveform representative of the optical signal variation generated by chained EDFAs within a longreach PON upstream link. In comparison with the approach of creating the power transients directly through a chain of EDFAs as implemented in [5], the approach used here has the advantage of varying the amplitude of the transients, which corresponds to a change in the number of chained EDFAs, by simply scaling the waveforms that drives the SOA. Moreover, the generation of the emulated optical transients can be synchronized with the optical bursts transmitted by the ONUs in order to analyse the effect of these transients as a function of their position within the upstream bursts. In particular, the OLT FPGA provides the electrical trigger for the waveform generator and controls the ONUs through the downstream protocol.

The waveform used for reproducing the profile of the residual optical transient has been measured at the output of a chain of 5 gain-stabilized EDFAs using a continuous-wave probe channel at $1550.12 \mathrm{~nm}$ when the background channels (between $1546.12-1563.85 \mathrm{~nm}$ ) have been turned on ("Add Event") and off ("Drop Event") with a period of $10 \mu$ s. These background channels are emulated by employing amplified spontaneous emission (ASE) which is generated by an EDFA before being filtered and flattened using a wavelength selective switch (WSS) and modulated using SOAs to emulate the typical burst mode power variations of upstream traffic as

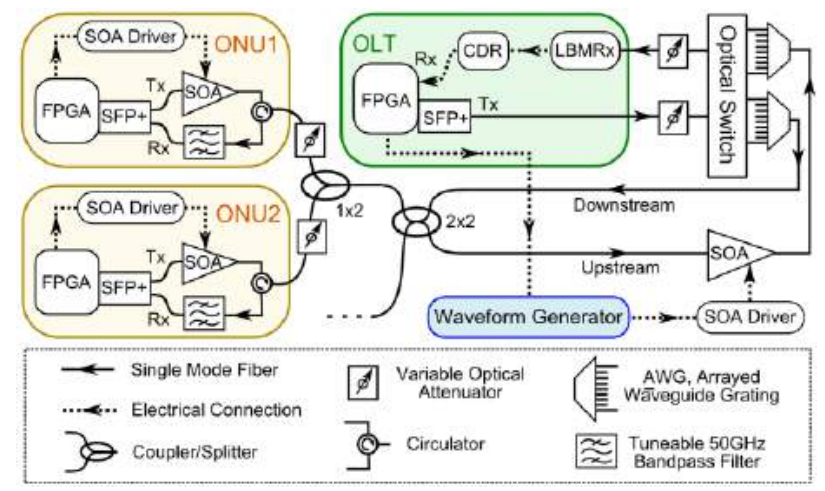

Fig. 2. PON testbed for EDFA transient emulation. 


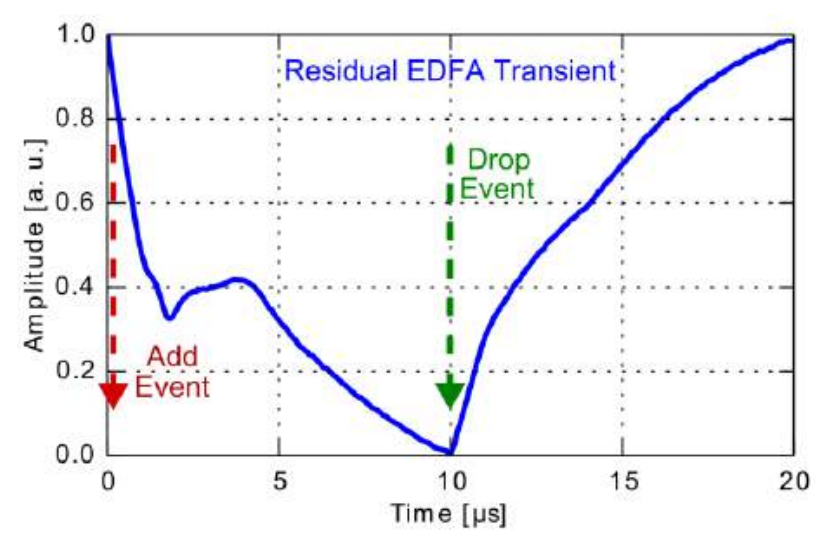

Fig. 3. Measured residual optical transient for an upstream optical link based on a chain of 5 gain-stabilized EDFAs with background traffic turned on ("Add Event") and off ("Drop Event") every $10 \mu \mathrm{s}$.

described in [5]. The measured optical transient profile is plotted in Fig. 3. This profile is the result of the intrinsic gain dynamics of the EDFA coupled with the fast automatic gain stabilization inside the EDFA modules, which can lead to a rather complex shape as shown in Fig. 3. For a more detailed analysis of the dynamics involved we refer the interested reader to [12].

\section{BURST-MODE TRANSIENT ANALYSIS}

The burst-mode FEC performance has been analysed as function of the amplitude of the emulated optical transients and their delay with respect to the upstream bursts. In order to equally affect the two ONUs, the duration of the bursts transmitted by the ONUs has been fixed to approximately $5 \mu \mathrm{s}$, which is half of the period $(10 \mu \mathrm{s})$ of add-and-drop events. This scenario is schematically depicted in Fig. 4, where the duration of the sketched transients is compared with the duration of the time-multiplexed upstream bursts of the ONUs. From this figure one can see that the transient delay needs to be varied between 0 and $10 \mu \mathrm{s}$ in order to analyse all possible alignments between the optical transients and the optical bursts.

These considerations have led us to the characterization presented in Fig. 5, where the measured pre- and post-FEC BER for the two ONUs is plotted with varying transient delay and with a transient amplitude of 5.2dB. This particular value has been experimentally determined in order to be close to the minimum transient amplitude before the FEC degradation. In Fig. 5 the measured pre-FEC BER of both ONUs always stays below the FEC threshold, hence the post-FEC BER of both ONUs is expected to be lower than 1e-12. However, for a transient delay close to 0 the post-FEC BER of ONU1 clearly raises above 1e-12, while for a transient delay close to $5 \mu$ s the post-FEC BER of ONU2 increases. This degradation of the FEC performance occurs because the optical transients introduce correlated and localized errors within the bursts. Hence, Fig. 5 demonstrates that, in upstream PON links affected by optical transients, the verification of a pre-FEC BER lower than the FEC threshold is not sufficient for assuring the correct FEC performance. In these cases, the post-FEC BER must also be characterized in order to verify the correct FEC behaviour.

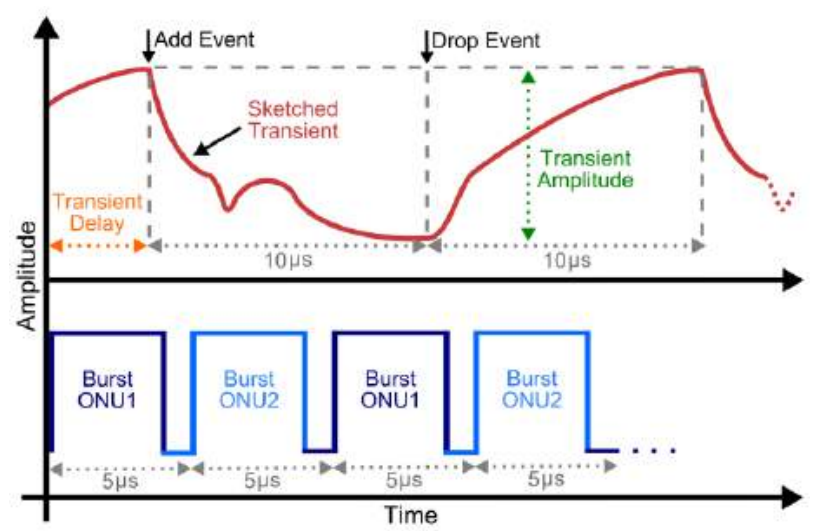

Fig. 4. Duration of sketched emulated transients compared with duration of upstream bursts transmitted by two time-multiplexed ONUs.

The measurements presented in Fig. 5 have been used to identify the following transient delays which represent the two worst cases for the FEC degradation: $0.1 \mu$ s for ONU1 and $5.2 \mu \mathrm{s}$ for ONU2. These two cases, which correspond to an "Add Event" aligned with the start of the burst, have been analysed in Fig. 6 (a) and (b) respectively, where the measured pre- and post-FEC BER of both ONUs has been plotted as a function of the emulated transient amplitude. In Fig. 6 the preFEC BER of both ONUs increases with the increase of the transient amplitude. This behaviour is expected considering that the increase of the transient amplitude leads to the increase of the number of errors within the burst. In Fig. 6 a margin of approximately $5 \mathrm{~dB}$ can be estimated by considering solely a pre-FEC BER below the FEC threshold. However, for transient amplitudes larger than $4 \mathrm{~dB}$ the post-FEC BER increases above 1e-12 with a pre-FEC BER lower than the FEC threshold, indicating the degradation of the FEC performance. The difference of approximately $0.3 \mathrm{~dB}$ between the margins measured for ONU1 and ONU2 is expected to be within the fabrication tolerances of the different SFP+ transmitters employed in the ONUs. The difference of approximately $1 \mathrm{~dB}$ between the pre- and post-FEC margins could be particularly relevant when designing PON architectures within the strict optical power budget of the current PON standards. Finally, this work also demonstrates the relevance of characterizing the

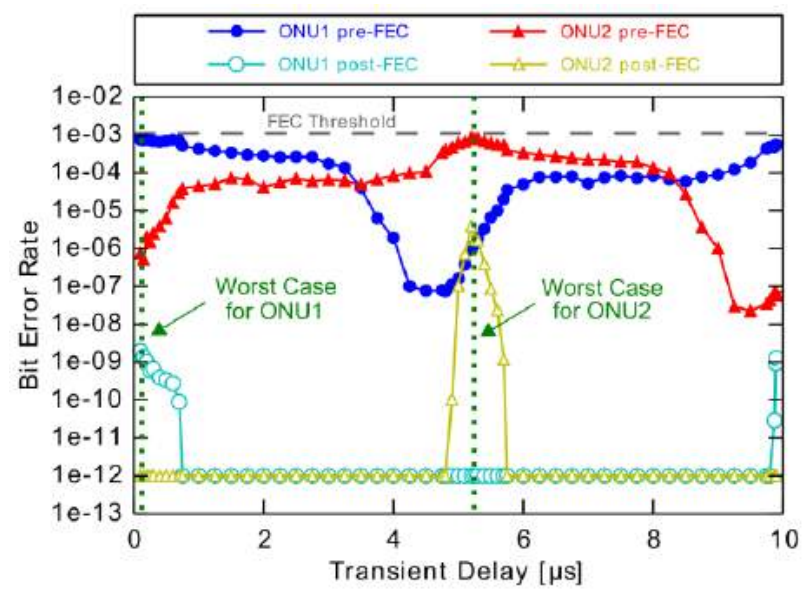

Fig. 5. Measured burst-Mode FEC performance with fixed transient amplitude of $5.2 \mathrm{~dB}$ and with transient delay varied with respect to the upstream bursts. 

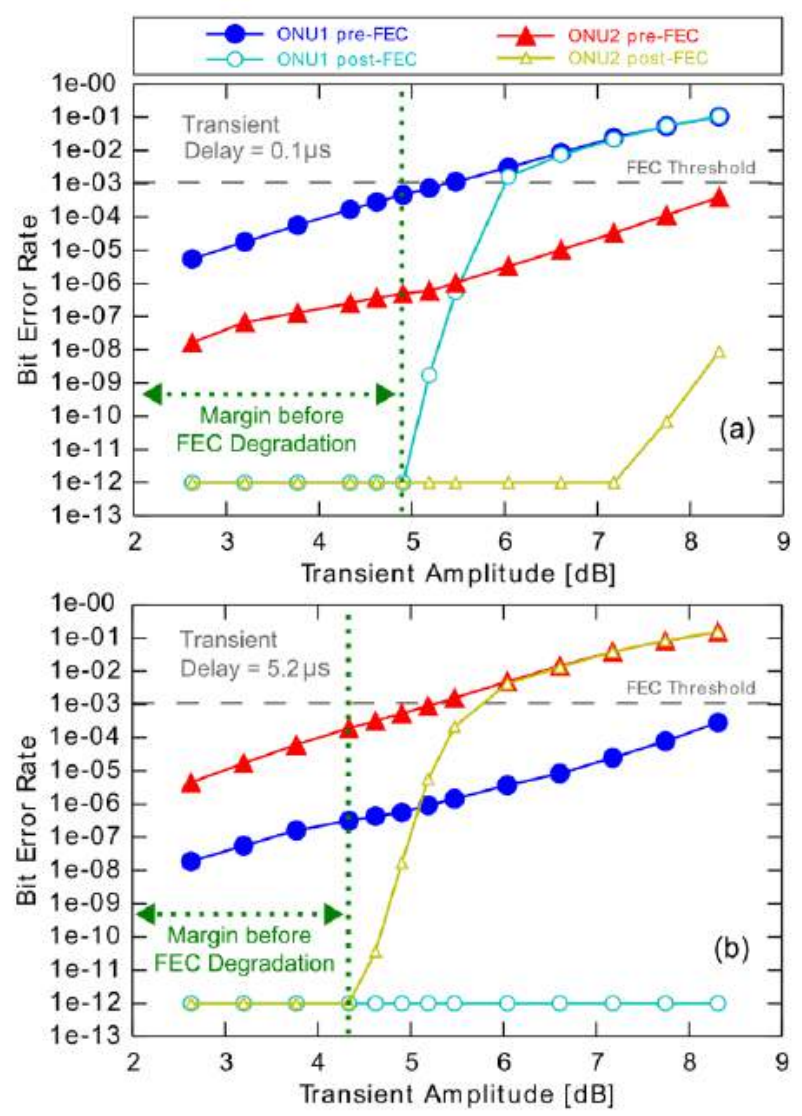

Fig. 6. Measured burst-mode FEC performance for two ONUs when the amplitude of the emulated EDFA transients is varied with transient delays of $0.1 \mu \mathrm{s}(\mathrm{a})$ and $5.2 \mu \mathrm{s}(\mathrm{b})$.

post-FEC BER in PON upstream channels as a function of the temporal distribution of the errors within the burst [8].

\section{CONCLUSIONS}

The analysis of burst-mode FEC performance within a PON upstream channel with emulated optical transients has been presented. These transients have been emulated in order to mimic the optical signal variation caused by added and dropped wavelength channels within long-reach PON upstream links with chained EDFA-based ANs. The amplitude of the optical transients and their delay with respect to the bursts have been varied in order to investigate the robustness of the FEC. During this analysis, the emulated optical transients have degraded the FEC performance by introducing correlated and localized errors within the bursts. Two worst cases for the FEC performance have been analysed for the two ONUs, demonstrating a margin of approximately $4 \mathrm{~dB}$ for the transient amplitude before the FEC degradation. This work has also demonstrated the importance of characterizing the post-FEC BER in PON upstream channels affected by optical transients, which can lead to the degradation of the FEC performance while leaving the pre-FEC BER below the FEC threshold.

\section{REFERENCES}

[1] D. Nesset, "PON roadmap [invited]," IEEE/OSA Journal of Optical Communications and Networking, vol. 9, no. 1, pp. A71-A76, 2017.

[2] M. Ruffini, M. Achouche, A. Arbelaez, R. Bonk, A. Di Giglio, N. J. Doran, M. Furdek, R. Jensen, J. Montalvo, N. Parsons, T. Pfeiffer, L. Quesada, C. Raack, H. Rohde, M. Schiano, G. Talli, P. Townsend, R. Wessaly, L. Wosinska, X. Yin, and D. B. Payne, "Access and metro network convergence for flexible end-to-end network design [invited]," IEEE/OSA Journal of Optical Communications and Networking, vol. 9 , no. 6, pp. 524-535, 2017.

[3] G. Talli, F. Slyne, S. Porto, D. Carey, N. Brandonisio, A. Naughton, P. Ossieur, S. McGettrick, C. Blümm, M. Ruffini, D. Payne, R. Bonk, T. Pfeiffer, N. Parsons, and P. Townsend, "SDN enabled dynamically reconfigurable high capacity optical access architecture for converged services," Journal of Lightwave Technology, vol. 35, no. 3, 2017.

[4] G. Talli, S. Porto, D. Carey, N. Brandonisio, A. Naughton, P. Ossieur, F. Slyne, S. McGettrick, C. Blum, M. Ruffini, D. Payne, R. Bonk, T. Pfeiffer, N. Parsons, and P. Townsend, "Demonstration of SDN enabled dynamically reconfigurable high capacity optical access for converged services," Optical Fiber Communications Conference (OFC), Postdeadline Paper Th5B.1, 2016.

[5] D. Carey, N. Brandonisio, S. Porto, A. Naughton, P. Ossieur, N. Parsons, G. Talli, P. Townsend, "Dynamically reconfigurable TDMDWDM PON ring architecture for efficient rural deployment," European Conference on Optical Communication (ECOC), pp. 692-694, 2016.

[6] ITU-T G.987.3, "10-gigabit-capable passive optical networks (XGPON): transmission convergence (TC) layer specification," 2014

[7] ITU-T G.989.2, "40-gigabit-capable passive optical networks 2 (NGPON2): physical media dependent (PMD) layer specification," 2014.

[8] N. Brandonisio, S. Porto, D. Carey, P. Ossieur, G. Talli, N. Parsons, and Paul Townsend, "Forward error correction analysis for $10 \mathrm{~Gb} / \mathrm{s}$ burstmode transmission in TDM-DWDM PONs," Optical Fiber Communications Conference (OFC), Paper Th2A.28, 2017.

[9] E. I. de Betou, E. Mobilon, B. Angeli, P. Öhlen, A. Lindström, S. Dahlfort, E. Trojer, "Upstream FEC performance in combination with burst mode receivers for next generation $10 \mathrm{Gbit} / \mathrm{s}$ PON," European Conference on Optical Communication (ECOC), Paper Mo.2.B.5, 2010.

[10] P. Ossieur, N. A. Quadir, S. Porto, C. Antony, W. Han, M. Rensing, P. O'Brien, and P. D. Townsend, "A 10Gb/s linear burst-mode receiver in 0.25 um SiGe:C BiCMOS," IEEE Journal of Solid-State Circuits, vol. 48, no. 2, pp. 381-390, 2013.

[11] S. Porto, C. Antony, A. Jain, D. Kelly, D. Carey, G. Talli, P. Ossieur, and P. D. Townsend, "Demonstration of 10Gbit/s burst-mode transmission using a linear burst-mode receiver and burst-mode electronic equalization [invited]," IEEE/OSA Journal of Optical Communications and Networking, vol. 7, no. 1, pp. A118-A125, 2015.

[12] A. Kaszubowska-Anandarajah, R. Oberland, E. Bravi, A. Surpin, O. Aharoni, U. Ghera, R. Giller, E. Connolly, E. K. MacHale, M.Todd, G. Talli, and D. McDonald, "EDFA transient suppression in optical burst switching systems," International Conference on Transparent Optical Networks (ICTON), Paper Mo.B2.4, pp. 1-4, 2012. 